\title{
Long exposure of non-cytotoxic concentrations of methylselenol suppresses the invasive potential of $\mathrm{B} 16 \mathrm{~F} 10$ melanoma
}

\author{
AEYUNG KIM ${ }^{1,2}$, JI-YONG JUNG ${ }^{1}$, MINSIK SON $^{3}$, \\ SANG-HAN LEE ${ }^{3}$, JONG-SEOK LIM ${ }^{2}$ and AN-SIK CHUNG ${ }^{1}$ \\ ${ }^{1}$ Department of Biological Sciences, Korea Advanced Institute of Science and Technology, \\ Daejeon 305-701; ${ }^{2}$ Department of Biological Science and the Research Center for \\ Women's Diseases, Sookmyung Women's University, Seoul 140-742; ${ }^{3}$ Department of Food \\ Science and Biotechnology, Kyungpook National University, Daegu 702-701, Korea
}

Received January 30, 2008; Accepted March 26, 2008

DOI: $10.3892 /$ or_00000042

\begin{abstract}
To assess the inhibitory effects of methylselenol on the invasion of murine B16F10 melanoma cells, we carried out in vivo and in vitro experiments using Se-methylselenocysteine (Se-MSC) and selenomethionine (SeMet), respectively. In an animal experiment, the supplementation of drinking water with Se-MSC (4 ppm Se) led to a significant increase in Se levels in the lung, liver and serum in mice. Mice given a mash diet or water supplemented with Se-MSC (2, 4 and 6 ppm Se in the mash diet, and 2 and 4 ppm Se in the drinking water) displayed an almost completely diminished pulmonary metastasis of B16F10 melanoma cells and an enhanced survival, compared to the control mice which were given a basal diet. Treatment with non-cytotoxic concentrations of SeMet $(2.5,5$ and $10 \mu \mathrm{M}$ plus $0.02 \mathrm{U} / \mathrm{ml}$ METase, methioninase) induced a substantial decrease in the expression of integrin $\alpha \mathrm{v} \beta 3$, the $\mathrm{FN}$ receptor and adhesion ability to vitronectin (VN) and fibronectin (FN) in B16F10 melanoma cells. Moreover, these compounds suppressed gelatinase activity, invasive ability and wound migration in the culture system. SeMet-METase prevented the conversion of proMMP-9 to its active form and decreased pro-MMP-2 activities in a zymogram. The pre-treatment of B16F10 melanoma cells with SeMet-METase led to a decrease in pulmonary metastasis and extended survival in mice injected with tumor cells. Collectively, our results indicate that integrin expression
\end{abstract}

Correspondence to: Dr An-Sik Chung, Department of Biological Sciences, Korea Advanced Institute of Science and Technology, Daejeon 305-701, Korea

E-mail: aschung@kaist.ac.kr

Abbreviations: SeMet, selenomethionine; METase, methioninase; Se-MSC, methylselenocysteine; VN, vitronectin; FN, fibronectin; MMP, matrix metalloproteinase; ECM, extracellular matrix

Key words: methylselenol, cell adhesion, integrin, chemoprevention, melanoma is crucial in promoting the metastatic phenotype in murine B16F10 melanoma cells by supporting specific adhesive and invasive properties, suggesting that Se-MSC effectively reduces the metastasis of $\mathrm{B} 16 \mathrm{~F} 10$ melanoma cells as a nutritional adjuvant. Methylselenol may also contribute to the suppression of integrin expression.

\section{Introduction}

Melanoma is the most fatal form of skin cancer, and its incidence in the USA and Australia has rapidly increased over the past decades. Since malignant melanoma has significant metastatic potential and is relatively resistant to current chemotherapeutic agents, the rate of mortality is high, despite extensive educational sunlight protection programs. Drugs that inhibit proliferation and induce apoptosis of cancer cells in the clinic are often associated with toxic side-effects, owing to the non-selective killing of proliferating cells. Thus, novel clinical approaches need to be developed to improve the efficiency of treatment of disseminated metastatic melanoma (1-3).

Metastasis, one of the major causes of cancer mortality, is a multi-step process which includes adhesion to the extracellular matrix (ECM), invasion of lymph and blood vessels and migration. Early events in cancer cell invasion involve cellcell or cell-ECM adhesion, and these are usually mediated by a number of cell surface molecules. Integrins are composed of various $\alpha$ and $\beta$ subunits, and mediate cell adhesion to several ECM proteins, including vitronectin (VN) and fibronectin (FN). Integrin-dependent interactions with ECM proteins play an important role in modulating the proliferation of tumor cells and their ability to release ECM-degrading enzymes, including MMP-2 (gelatinase A/72 kDa type IV collagenase), MMP-9 (gelatinase B/92 kDa type IV collagenase) and urokinase type plasminogen activator (uPA). Therefore, blocking the integrin expression and disrupting adhesion may be an effective strategy for preventing the proliferation and metastasis of melanomas (4-11). Earlier studies have shown that dietary supplementation with selenium reduces lung metastasis of melanoma cells, and selenium yeast inhibits the spread of Lewis lung carcinoma cells in mice (12-14). These 
findings collectively suggest that a nutritional adjuvant containing selenium may be beneficial in reducing metastasis.

Selenium is an essential trace element with dramatic variations in geographical abundance. Selenium deficiency is related to the occurrence of specific chronic diseases, including cancer in humans, while adequate selenium intake is associated with the lower incidence of certain cancers in humans (15-17). The addition of specific selenium compounds to cell culture systems or supplementation into animal diets leads to reduced cell proliferation and apoptosis in vitro, as well as a decreased incidence and severity of cancer in vivo $(12,13,18)$. It is proposed that the effects of these compounds on tumor cell proliferation and the onset of cancer in animals are dependent on the dose and chemical composition (19-21). Several groups are involved in the search for active metabolites that appear crucial for selenium-induced cancer chemoprevention, and the identification of the least toxic form that provides reliable chemopreventive activity $(22,23)$. There is ample evidence that the chemical transformation of selenium to a monomethylated metabolite is an important step in chemoprevention. Current experimental evidence indicate that methylselenol $\left(\mathrm{CH}_{3} \mathrm{SeH}\right)$ is the selenium metabolite responsible for the dietary chemoprevention of cancers. The addition of methioninase (METase) to selenomethionine (SeMet) or ß-lyase to Se-methylselenocysteine (Se-MSC) rapidly generates significant amounts of methylselenol, with anticarcinogenic activity comparable to that of selenite or methylseleninic acid (MSeA) (19,24-29). Se-MSC and SeMet, the organic dietary forms of selenium, are bioavailable with low toxicity, and are thus better targets for chemoprevention than sodium selenite $\left(\mathrm{Na}_{2} \mathrm{SeO}_{3}\right)(19,20,30,31)$. While several studies have focused on the effects of selenium on primary tumor development and growth, the molecular processes involved in melanoma cell adhesion and invasion, and their modulation by selenium compounds have yet to be established.

Since adhesion is involved in most of the intermediate steps of the metastatic cascade, we examine the correlation of a decreased integrin expression following SeMet-METase treatment with invasion and pulmonary metastasis. The effects of diet supplementation with Se-MSC on the pulmonary metastasis of B16F10 melanoma cells and the survival of C57BL/6J mice in animal experiments are also investigated.

\section{Materials and methods}

Mice and cell line. Four-week-old female C57BL/6J mice purchased from the BioModel System Park (Daejeon, Korea) were housed under specific pathogen-free conditions in a barrier facility with 12-h light/dark cycles. The murine B16F10 melanoma cell line was maintained as a monolayer culture in RPMI-1640 medium (Gibco/Invitrogen, Carlsbad, CA) supplemented with $10 \mathrm{mM}$ HEPES, $50 \mathrm{mg} / 1$ gentamicin (Life Technologies, Inc., Rockville, MD) and 10\% heat-inactivated fetal bovine serum (FBS, Gibco/Invitrogen) in a humidified $5 \% \mathrm{CO}_{2}$ incubator.

Antibodies and chemicals. SeMet and Se-MSC were acquired from PharmaSe, Inc. (Lubbock, TX) and selenite from Sigma (St. Louis, MO). L-methionine $\gamma$-Lyase (designated METase) was purchased from Wako Pure Chemical Industries, Ltd. (Osaka, Japan). R-phycoerythrin (R-PE)-conjugated rat antimouse CD51 (integrin $\alpha \mathrm{v}$ chain) and anti-mouse CD61 (integrin $B 3$ chain), purified anti-fibronectin receptor and anti-mouse CD51 were all purchased from BD Bioscience Pharmingen (Bedford, MA). Bovine plasma FN was purchased from Sigma and human plasma VN from Alexis (San Diego, CA).

Gelatin zymography. The enzymatic activities of MMP-2 and -9 were assayed by gelatin zymography. A serum-free culture medium was collected after incubation with various concentrations of SeMet-METase or selenite, for $48 \mathrm{~h}$. Aliquots of the supernatants were concentrated using a Centricon (Amicon, Inc., Beverly, MA), and equivalent volumes of the culture supernatant fractions were electrophoresed on a $10 \%$ SDS-polyacrylamide gel containing $0.1 \%$ gelatin. Gels were washed twice with a washing buffer $(50 \mathrm{mM}$ Tris- $\mathrm{HCl}, \mathrm{pH} 7.5,100 \mathrm{mM} \mathrm{NaCl}$ and 2.5\% Triton X-100), followed by brief rinsing in this washing buffer, excluding Triton $\mathrm{X}-100$. The gels were then treated with an incubation buffer $(50 \mathrm{mM}$ Tris- $\mathrm{HCl}$, pH 7.5, $150 \mathrm{mM} \mathrm{NaCl}, 10 \mathrm{mM}$ $\mathrm{CaCl}_{2}, 0.02 \% \mathrm{NaN}_{3}$ and $\left.1 \mu \mathrm{M} \mathrm{ZnCl}_{2}\right)$ at $37^{\circ} \mathrm{C}$ for $18-36 \mathrm{~h}$, stained $(0.05 \%$ Coomassie blue, $10 \%$ isopropanol and $10 \%$ acetic acid) and destained (10\% isopropanol and 10\% acetic acid). MMPs were detected as transparent bands on the blue background of Coomassie blue-stained slab gels.

In vitro tumor cell invasion assay. The in vitro invasion assay was performed using the matrigel-coated transwell system. Briefly, after filling the lower chambers with RPMI medium plus $10 \%$ FCS as a chemoattractant, transwell (8- $\mu \mathrm{m}$ pore), coated with $20 \mu \mathrm{l}$ of a 1:2 mixture of matrigel:RPMI, was assembled as the intervening invasive barrier on a 24-well plate. Cells $\left(5 \times 10^{4}\right)$ were suspended in a serum-free RPMI medium, added to the upper part of the chamber and incubated for $18 \mathrm{~h}$ at $37^{\circ} \mathrm{C}$. The cells which remained on the upper surface of the filter were completely removed by wiping with a cotton swab, and those on the lower surface of the filters were fixed in methanol, and stained with hematoxylin and eosin. The cells invading the lower surface of the filters were counted manually under a light microscope. The invasive potential of untreated control cells was normalized to $100 \%$ invasion, and that of treated cells was determined as a percentage of the control.

Wound-healing assay. B16F10 cells were pre-incubated with $25 \mu \mathrm{g} / \mathrm{ml}$ mitomycin $\mathrm{C}$ for $30 \mathrm{~min}$. An injury line was made on the confluent monolayer of cells with a cell scraper.

After rinsing with PBS, cells were allowed to migrate in the presence of selenium compounds. Migration was observed with a phase-contrast microscope and photographs were obtained at the indicated time-points.

Flow cytometry. Cells were harvested, washed twice with PBS, and incubated in PBS containing 1\% FBS for 30 min at $4{ }^{\circ} \mathrm{C}$. Cells were collected by centrifugation and exposed to saturating concentrations of individual PE-conjugated antimouse $\alpha \mathrm{V}$ - or B3-integrin antibodies. After incubation on ice for $30 \mathrm{~min}$, cells were washed and the relative amounts of cell 
surface integrins were determined by the comparison of fluorescence emission intensities collected using FACSCalibur (Beckton-Dickinson, NJ).

Cell-adhesion assay on immobilized VN and FN. For cell-toECM adhesion assay, 24-well plates were coated with $10 \mu \mathrm{g} / \mathrm{ml}$ of VN or FN for $16 \mathrm{~h}$ at $4^{\circ} \mathrm{C}$, washed three times with cold PBS, coated with $3 \%$ bovine serum albumin for $1 \mathrm{~h}$ at $37^{\circ} \mathrm{C}$ and washed three times. Cells suspended in serum-free RPMI-1640 medium were plated on ECM-coated culture plates and incubated for $1.5 \mathrm{~h}$ at $37^{\circ} \mathrm{C}$ in a $5 \% \mathrm{CO}_{2}$ humidified atmosphere. Plates were washed three times with PBS to remove non-adherent cells and cells remaining attached to the plate were fixed and stained with a solution of $0.5 \%$ crystal violet $/ 2 \%(\mathrm{v} / \mathrm{v})$ ethanol in $100 \mathrm{mM}$ borate buffer $(\mathrm{pH} 9.0)$ at room temperature for $30 \mathrm{~min}$. Cells were lysed with $1 \%$ SDS, and spectrophotometric absorbance was measured at $592 \mathrm{~nm}$.

Experimental pulmonary metastasis assay. Mice were divided into eight groups, composed of 20 animals each. An additional group of the same strain of animals $(n=10$, designated normal) not subjected to selenium treatment or tumor injection was included in the analysis. Control mice were given a basal diet $(0.23 \mathrm{ppm} \mathrm{Se})$ and water $(0.00134 \mathrm{ppm})$ during the course of the experiment. A mash diet supplemented with various concentrations of Se-MSC (2, 4 and 6 ppm Se) was fed to four-week-old female C57BL/6J mice for 4 weeks. Thereafter, B16F10 melanoma cells $\left(3 \times 10^{5}\right.$ cells $/ 0.2 \mathrm{ml}$ PBS $)$ were injected intravenously into the lateral tail veins of C57BL/6 mice. Following the administration of cells, the mice were allowed to consume their respective diets for an additional 40 days. On day 20 of the post-tumor injection, five mice from each group were euthanized, their lungs removed and the extent of metastasis observed macroscopically. Drinking water supplemented with various concentrations of Se-MSC (2, 4 and $6 \mathrm{ppm} \mathrm{Se}$ ) was used during the above procedure. The survival rates of the 10 remaining mice in all groups were recorded over a 40-day period.

Determination of the Se level. To determine the Se concentration in mice tissues, serum, lung and liver samples were obtained after supplementation with $4 \mathrm{ppm}$ Se as Se-MSC for 4 weeks immediately prior to the tumor cell injection. Samples were analyzed by graphite furnace atomic absorption spectrophotometry (GFAAS, Analyst 880 Graphite, Perkin Elmer, USA). Se concentrations in the diet and drinking water were measured by hydride vapor-generated atomic absorption spectroscopy (HVG-AAS, Shimazu 6800, Japan).

Statistical analysis. Results are presented as mean \pm standard deviation (SD). All experiments were repeated at least three times, with documented reproducibility. Data were analyzed for statistical significance using Student's t-test. $\mathrm{P}<0.05$ was considered significant.

\section{Results}

Se-MSC intake has no toxic side-effects and increases the selenium level in lung, liver and serum. In our animal studies, the pulmonary metastasis of B16F10 melanoma and survival
C57BL/6J mice (female; 4 wks old)

Se level in lung, liver, and serum $(n=5)$ : Day 0

$\downarrow$ i.v injection of $\mathrm{B} 16 \mathrm{~F} 10$ cells $\left(3 \times 10^{5}\right.$ cells, $\left.\mathrm{n}=15\right)$ : Day 0

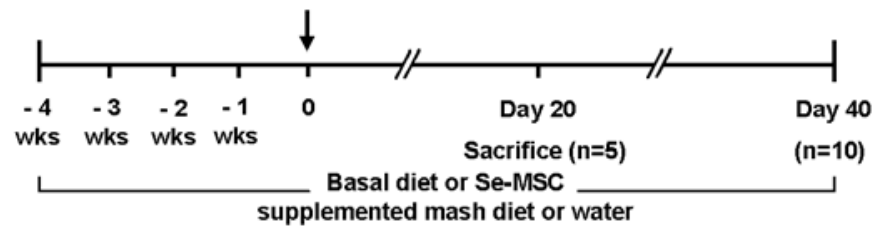

Figure 1. Experimental design. Four-week-old female C57BL/6J mice were fed a Se-MSC (2, 4 and 6 ppm Se)-supplemented diet or water. The control mice were fed a basal diet or water containing no additional selenium compounds. After 4 weeks, lung, liver and serum were collected from 5 randomized mice for assessment of the selenium concentration. To examine the effect of dietary selenium supplementation on the pulmonary metastasis of B16F10 melanoma cells and survival of mice, B16F10 melanoma cells were injected intravenously via the lateral tail vein after supplementation with selenium-containing or basal diet. On day 20 after the tumor injection, the pulmonary metastasis of B16F10 melanoma cells were macroscopically observed, and survival was checked every 2 days for 40 days after the tumor injection.

in $\mathrm{C} 57 \mathrm{BL} / 6 \mathrm{~J}$ mice were investigated after supplementation with Se-MSC (Fig. 1). In preliminary experiments, the mean amount of food and water intake was $\sim 3.92 \pm 0.78 \mathrm{~g} / \mathrm{mouse} /$ day and $5.23 \pm 0.85 \mathrm{ml} / \mathrm{mouse} /$ day, respectively. The Se concentration in the mash diet was $0.23 \mathrm{ppm}$, similar to the level suggested by the manufacturer $(0.22 \mathrm{ppm})$ and the water contained $0.0013 \mathrm{ppm}$ Se. Se-MSC was mixed with the mash diet or drinking water at non-toxic concentrations relative to the intake amount. Concentrations that induced side-effects, such as hair loss, were excluded from the main experiment. Mice were given a Se-MSC-containing mash diet or water for 4 weeks before the tumor injection and an additional 40 days thereafter. At the the time of the tumor injection, no significant differences in lung, liver, body weight (data not shown) or external appearance were evident among the groups. Supplementation with Se-MSC (4 ppm Se in water) for 4 weeks immediately before tumor inoculation led to increased $\mathrm{Se}$ levels in the liver, lung and serum in the mice, compared to the animals which were fed the control diet (Fig. 2A). The elevated Se levels in tissues reflected the dietary intake of selenium compounds.

Supplementation of Se-MSC reduces the metastasis potential of B16F10 melanoma cells and extends survival. On day 20 after tumor injection, mice from each group were euthanized so as to examine pulmonary metastasis. At this time, the mice being fed a basal diet displayed slow movement, and some were paralyzed from the waist down or had difficulty in breathing. Upon necropsy, no differences were found in the liver, kidney and heart weights among the groups and tumors were not evident in these organs by gross observation (data not shown). An intravenous injection of B16F10 melanoma cells into the lateral tail veins of mice, which were fed a basal diet, resulted in severe pulmonary metastasis, as shown in Fig. 2B. In contrast, mice administered with Se-MSC (2, 4 and $6 \mathrm{ppm}$ Se in the mash diet) only exhibited a slight pulmonary metastasis with an average nodule number of $5 \pm 2$. Moreover, some of these animals displayed no detectable 
A
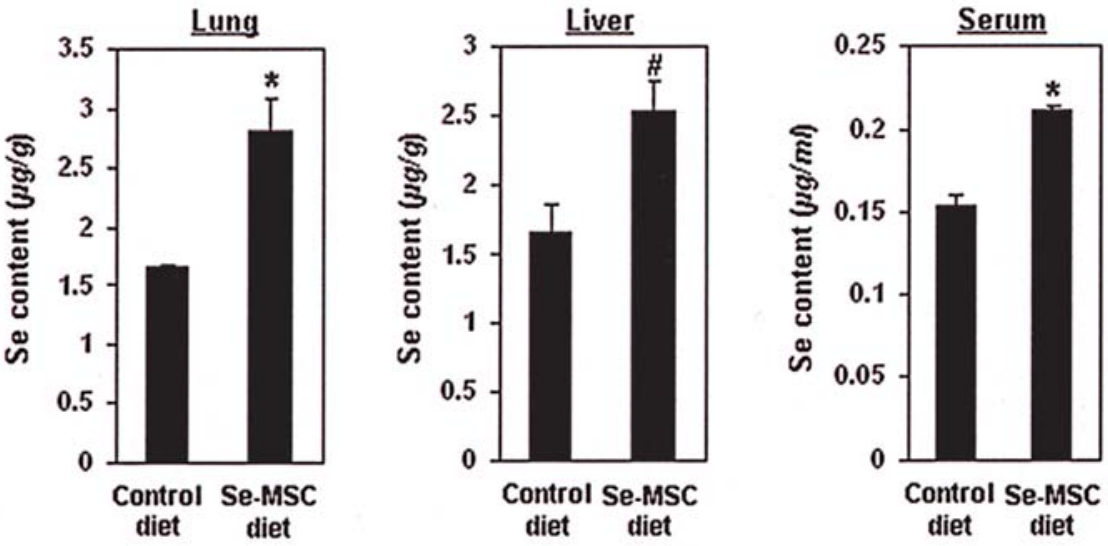

B
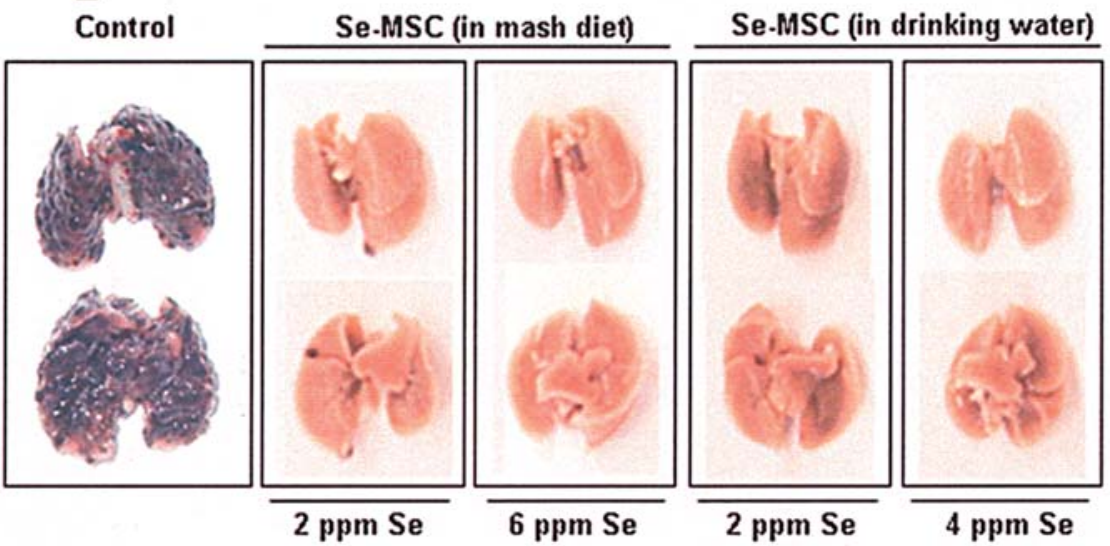

C
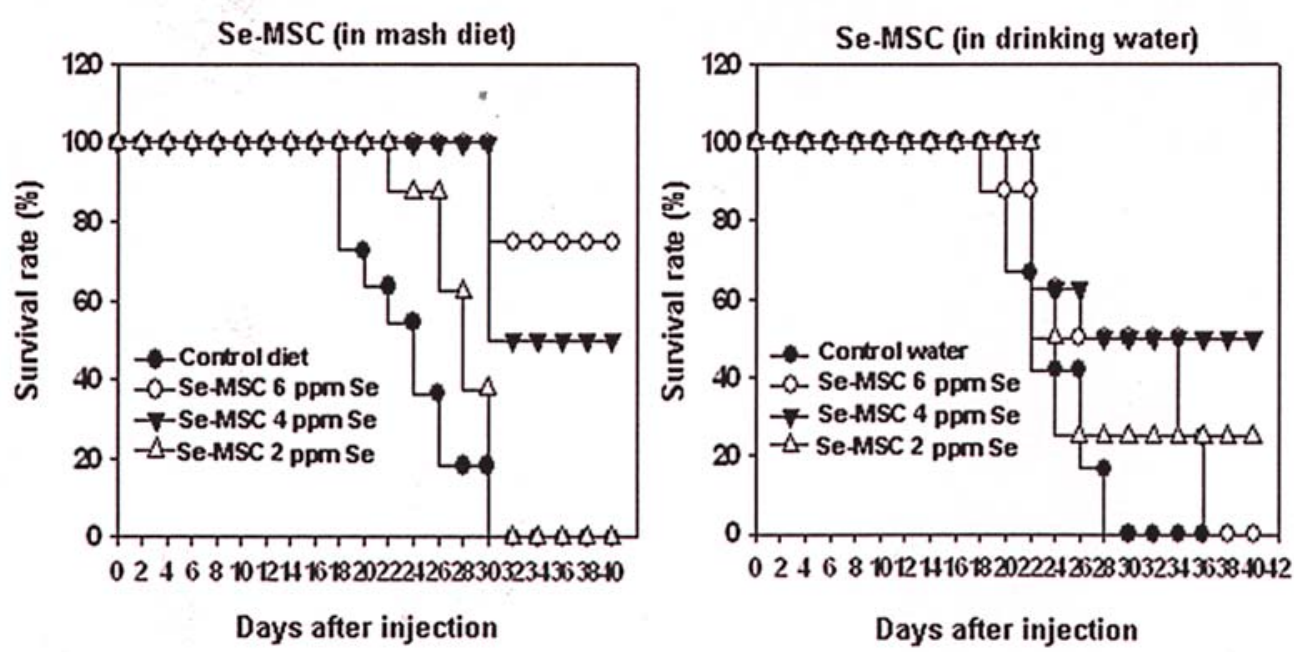

Figure 2. Effects of supplementation with Se-MSC on the pulmonary metastasis of B16F10 melanoma cells and survival of mice. (A) Lung, liver and serum Se concentration of mice fed a control diet and Se-MSC-supplemented diet. The values are expressed as means \pm SD; ${ }^{*}<<0.05$ and ${ }^{*} \mathrm{p}<0.01(\mathrm{values}$ are compared with the control). (B) On day 20 after the tumor injection, the mice were sacrificed, and the lung localization of cells was macroscopically observed. The black colony indicates metastasized B16F10 melanoma cells. The figure is a representative example of lungs from 3 individual experiments, with similar results. (C) Mice were examined for survival every 2 days, up to 40 days after the tumor injection. The survival study was repeated twice.

metastasis of the lung. Data on the survival rates at 40 days after the tumor injection revealed that supplementation with Se-MSC significantly prolonged the animal lifespan in a dose-dependent manner (Fig. 2C). The mean lifespan of mice fed on a basal diet was 24.7 days. In contrast, some mice administered with $6 \mathrm{ppm} \mathrm{Se}$ as Se-MSC in mash diet were still alive on day 60 after the tumor injection. Supplementation of the diet with high concentrations of selenium compounds (>10 ppm Se as Se-MSC) resulted in adverse side-effects (e.g. hair loss) or significantly elevated pulmonary metastasis, leading to accelerated death (data not shown). The drinking of water supplemented with Se-MSC led to the inhibition of pulmonary metastasis, as well as extension of the lifespan (Fig. 2B-C), although at $6 \mathrm{ppm}$ Se as Se-MSC in drinking 


\section{A}
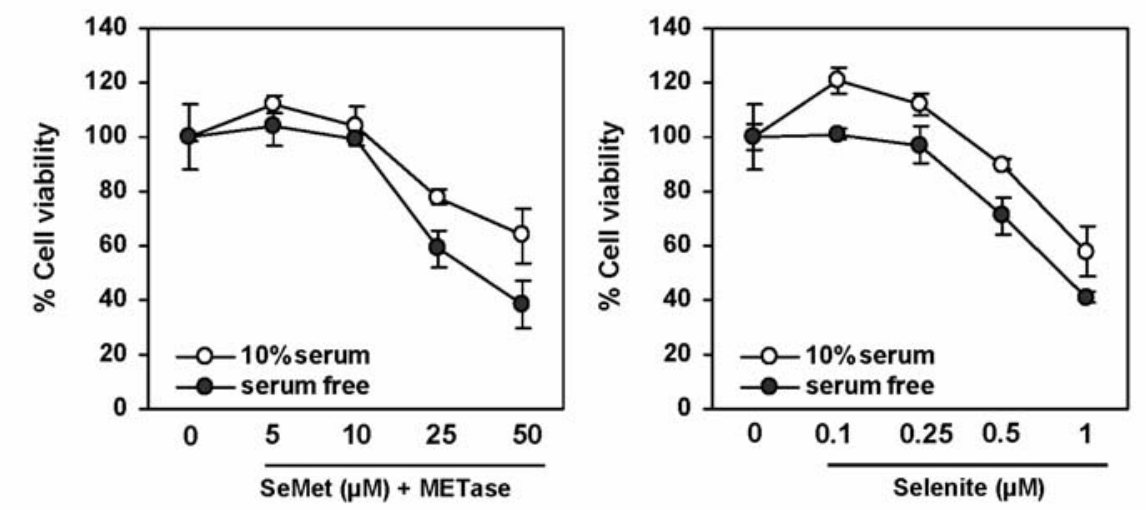

B

C
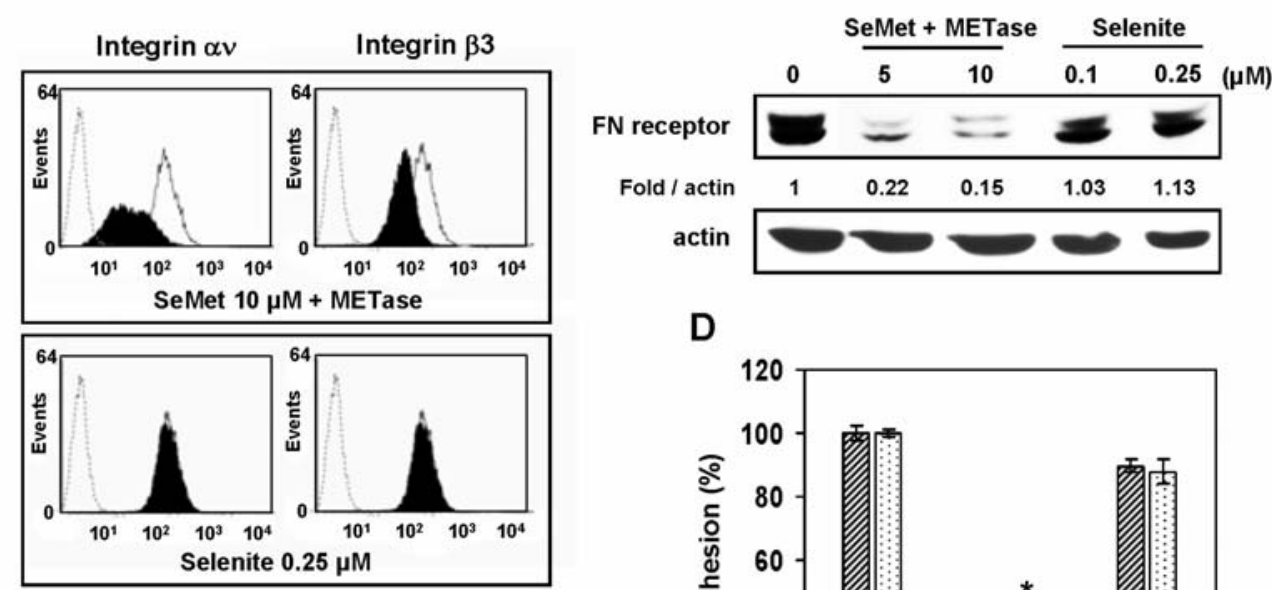

D

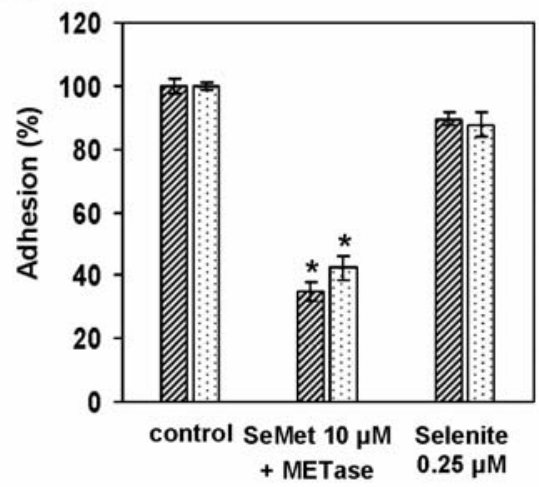

Figure 3. Effects of SeMet-METase, or selenite on integrin expression, and adhesion to VN or FN. (A) B16F10 cells were treated with SeMet-METase or selenite varying the concentration in the presence (white circle) or absence (black circle) of serum for $72 \mathrm{~h}$. Viability was measured by MTT assay, and expressed as means $\pm \mathrm{SD}$. (B) B16F10 cells were treated with non-toxic low concentrations of SeMet (10 $\mu \mathrm{M})$-METase or selenite $(0.25 \mu \mathrm{M})$ for $48 \mathrm{~h}$ in serum-free conditions. The expression of integrin $\alpha \mathrm{V}$ and $B 3$ was analyzed by flow cytometry. In the histogram, the dashed lines represent the basal integrin expression in B16F10 cells and the filled histograms indicate integrin expression upon SeMet-METase or selenite treatment. Results are presented as relative MFI (mean fluorescence intensity) $\pm \mathrm{SD} ;{ }^{*} \mathrm{p}<0.01$ (values are compared with the control). (C) Cells were treated with various concentrations of SeMetMETase or selenite for $48 \mathrm{~h}$ and lysed, and $50 \mu \mathrm{g}$ of each sample was subjected to Western blot analysis. (D) Adhesion to VN and FN was assayed using cells prepared as described above. After seeding to $\mathrm{VN}$ and $\mathrm{FN}$, cells were incubated for $1.5 \mathrm{~h}$, washed to remove non-adherent cells and those remaining attached to $\mathrm{VN}$ and $\mathrm{FN}$ were measured by crystal violet staining; ${ }^{\mathrm{p}}<0.01$ (values are compared with the control).

water, survival was not significantly prolonged (mean lifespan on the basal diet $=25.33$ days vs. 6 ppm Se as Se-MSC $=29.67$ days). Therefore, the oral supplementation with adequate concentrations of selenium in the form of Se-MSC via the mash diet or drinking water reduced lung metastasis and extended the lifespan of the animals.

Effect of methylselenol and selenite on the cell viability of B16F10 melanoma. To generate bioactive methylselenol from SeMet, METase was added at $0.02 \mathrm{U} / \mathrm{ml}$ to the cell culture medium as previously described (32). In our culture system, treatment with SeMet in the absence of METase had no influence on cellular morphology or viability (data not shown). In contrast, Se-MSC or L-methionine (Met), at levels up to $100 \mu \mathrm{M}$, did not influence cellular viability even when METase was also present (data not shown). This result suggests that the level of METase in the B16F10 cells is negligible, and the addition of METase to SeMet was essential in generating methylselenol in our experiment. Initially, the non-cytotoxic concentrations of SeMet-METase were determined by MTT assay in serum-free conditions and $10 \%$ serumcontaining media to exclude the possibility of apoptotic effects and ensure consistency with in vivo conditions. Selenite was used throughout the in vitro experiments as an inorganic selenium control. As shown in Fig. 3B, in the serum-free conditions and with media containing $10 \%$ serum, 
A

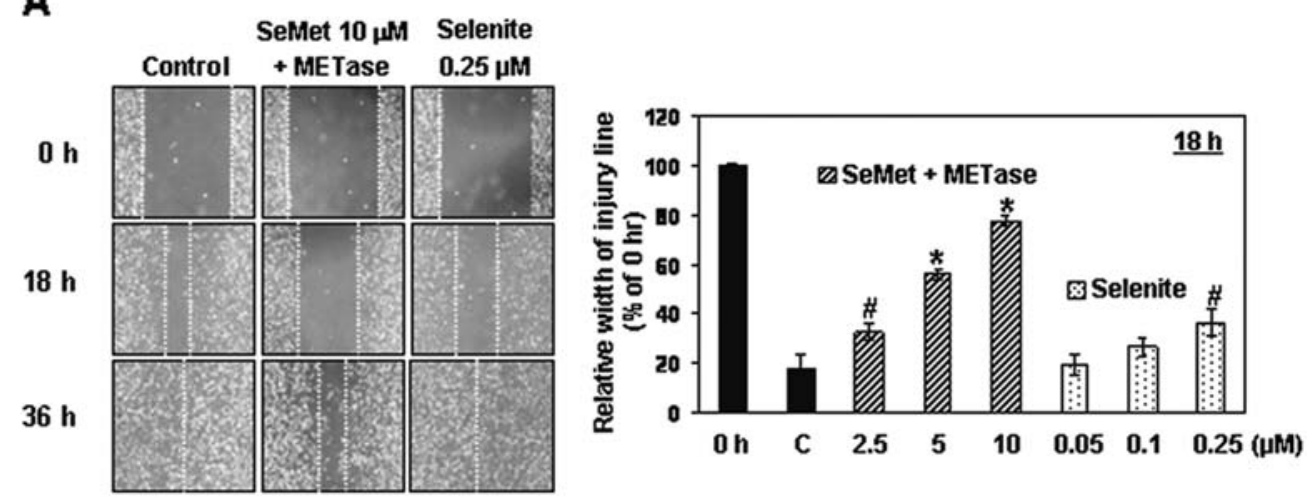

B
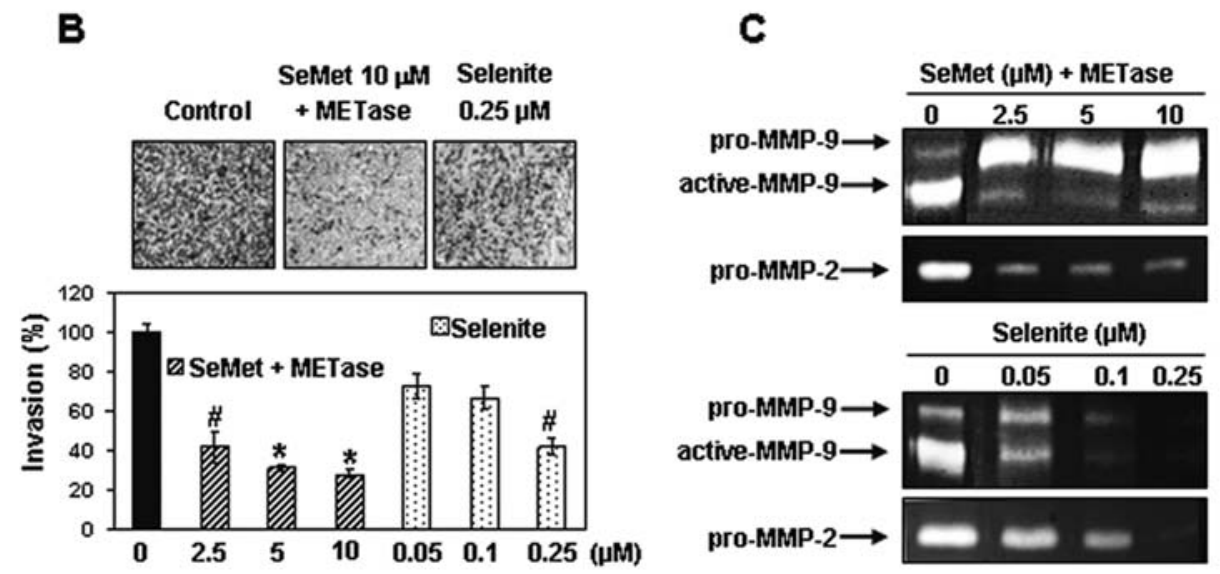

Figure 4. Effects of SeMet-METase, or selenite on the migration, invasive capacity and gelatinolytic activities of MMP-2 and -9 in B16F10 melanoma cells. (A) B16F10 cells were pretreated with $25 \mu \mathrm{g} / \mathrm{ml}$ of mitomycin C for $30 \mathrm{~min}$. An injury line was created on the confluent monolayer, washed with PBS and cells were cultured with various concentrations of selenium compounds in serum-free conditions. Cell migration was monitored with a phase-contrast microscope $(\mathrm{x} 40)$ at the indicated time-points. The width of the injury line was measured, and the means \pm SD of three independent experiments were plotted; ${ }^{\#} \mathrm{p}<0.05$ and ${ }^{*} \mathrm{p}<0.01$ (values are compared with the control). (B) Cells $\left(5 \times 10^{4}\right.$ cells) treated with SeMet $(2.5,5$ and $10 \mu \mathrm{M})-\mathrm{METase}$, or selenite $(0.05,0.1$ and $0.25 \mu \mathrm{M})$ for $48 \mathrm{~h}$ were plated onto the upper part of the transwell chamber coated with matrigel, and incubated for $18 \mathrm{~h}$. Invasive cells were determined, as described in Materials and methods. The results are expressed as means \pm SD of three experiments; ${ }^{*} \mathrm{p}<0.05$ and ${ }^{*} \mathrm{p}<0.01$ (values are compared with the control). (C) Cells were incubated in serum-free media containing non-cytotoxic concentrations of SeMet $(2.5,5$ and $10 \mu \mathrm{M})$-METase and selenite $(0.05,0.1$ and $0.25 \mu \mathrm{M}$ ) for $48 \mathrm{~h}$. A conditioned medium was collected, concentrated using Centricon and subjected to gelatin for MMP-2 and - 9 zymography for the detection of secreted proteinase. The results are representative of three independent experiments.

$>25 \mu \mathrm{M}$ SeMet-METase or $0.5 \mu \mathrm{M}$ selenite, showed cytotoxicity. To ensure MTT assay, we performed a trypan blue dye exclusion assay and the results were similar to those of MTT assay (data not shown). Accordingly, we employed SeMet or selenite within a non-cytotoxic concentration for all subsequent in vitro experiments.

Methylselenol reduces the surface expression of integrin $\alpha v$ and $\beta 3$, and inhibits adhesion to $V N$ and $F N$. In a previous study, we showed that B16F10 melanoma cells express substantial amounts of integrins, including $\alpha \mathrm{V}$ and $\beta 3$, and treatment with concentrations of SeMet (above $50 \mu \mathrm{M}$ )METase $(0.02 \mathrm{U} / \mathrm{ml})$ for 3-6 h decreases surface integrins and cell viability (33). In the present experiments, B16F10 melanoma cells were treated with non-cytotoxic concentrations of SeMet-METase for long periods of time (48-72 h). Treatment with $10 \mu \mathrm{M}$ SeMet-METase induced a $>50 \%$ decrease in the surface expression of integrins $\alpha \mathrm{V}$ and $B 3$ (Fig. 3B), and a decreased FN receptor protein level (Fig. 3C). In contrast, treatment with selenite did not alter the surface expression of integrin $\alpha \mathrm{v} B 3$ and FN receptor levels. Since the primary ligands for $\alpha \mathrm{vB} 3$ are $\mathrm{FN}$ and $\mathrm{VN}$, untreated B16F10 melanoma cells exhibited an enhanced adhesion to FN and VN with increasing incubation times. Compared to the untreated B16F10 melanoma cells, SeMet-METase-treated cells significantly inhibited cell adhesion to FN or VN (Fig. 3D), but selenite-treated cells did not cause inhibition. Thus, it is proposed that a reduced integrin expression breaks the linkage between cells and $\mathrm{VN}$ or FN, abrogates the correct cytoskeletal organization required for melanoma metastasis and may contribute to a more potent anti-cancer effect.

Methylselenol inhibits migration, invasive and gelatinolytic ability of B16F10 melanoma cells. Migrative capacity is an essential prerequisite for cell invasion through the basement membrane. Accordingly, we assessed the effects of selenium compounds on migration using a wound-healing assay. As shown in Fig. 4A, B16F10 melanoma cells migrated to the wound, leading to almost complete healing within $36 \mathrm{~h}$, while treatment with $10 \mu \mathrm{M}$ SeMet-METase led to $>50 \%$ inhibition 
A

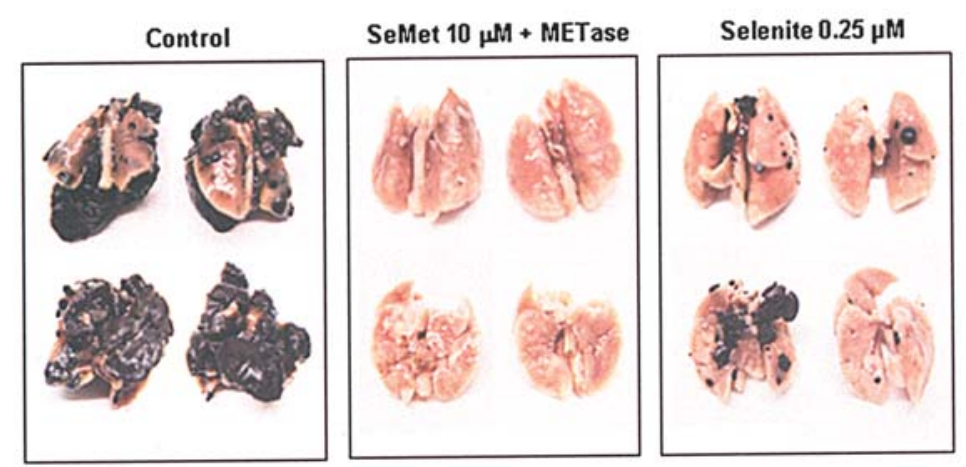

B

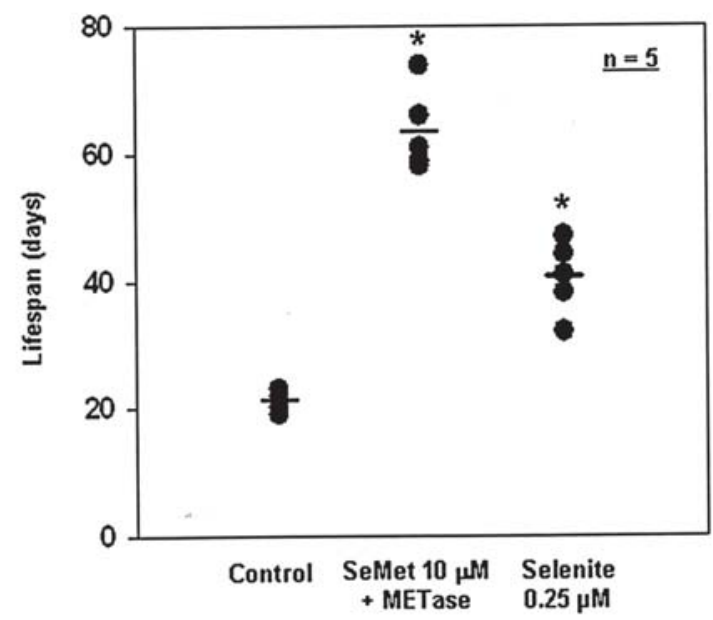

Figure 5. In vivo pulmonary metastasis experiment. (A-B) Cells treated with SeMet (10 $\mu \mathrm{M})$-METase or selenite ( $0.25 \mu \mathrm{M})$ for $48 \mathrm{~h}$ were counted, and $3 \mathrm{x} 10^{5}$ viable B16F10 melanoma cells were injected into the lateral tail vein. On day 20, lungs from the mice were observed macroscopically, and the lifespan was examined. In Fig. 5B, the bars represent the mean lifespan of each group; ${ }^{*}$ p $<0.01$ (values are compared with the control).

in cell migration $(18 \mathrm{~h})$. Dose-dependent inhibitory effects on cell migration were additionally observed after treatment for $18 \mathrm{~h}$. Treatment with $0.25 \mu \mathrm{M}$ selenite also inhibited cell migration (18 h), but was less efficient than SeMet-METase treatment. Consistent with these results, a transwell migration assay revealed a dose-dependent decrease in cell migration (data not shown). The in vitro invasion of B16F10 melanoma cells through a matrigel-coated filter was significantly reduced in the presence of $10 \mu \mathrm{M}$ SeMet-METase or $0.25 \mu \mathrm{M}$ selenite ( $>80 \%$ inhibition with SeMet-METase or $>50 \%$ with selenite, respectively, Fig. 4B). Matrix-degrading proteinases are essential to allow for the migration and invasion of cancer cells for metastasis. To analyze the direct effects of selenium compounds on gelatinolytic activity, B16F10 melanoma cells were incubated with increasing concentrations of SeMetMETase or selenite. The secretion of gelatin-degrading MMPs was analyzed by gelatin zymography. As shown in Fig. 4C, B16F10 melanoma cells constitutively secreted the latent proform of MMP-2 (72 kDa, gelatinase A) and the active form of MMP-9 (88 kDa, gelatinase B) into culture supernatants. SeMet-METase and selenite markedly decreased the gelatinolytic activity of proform MMP-2. In addition, SeMetMETase prevented the conversion of proform MMP-9 to its active form, while selenite decreased the gelatinolytic activities of the active and proform MMP-9. Based on the results, we propose that non-cytotoxic concentrations of SeMet-METase and selenite inhibit the gelatinolytic activities of MMP-2 and -9 in a different way.

Methylselenol reduces the in vivo metastatic potential of B16F10 melanoma cells. For the in vivo experiment, C57BL/6J mice were inoculated intravenously, via the lateral tail vein, with B16F10 melanoma cells treated with $10 \mu \mathrm{M}$ SeMetMETase or $0.25 \mu \mathrm{M}$ selenite for $48 \mathrm{~h}$. Cells treated with selenium compounds showed neither a decrease in cell viability nor apoptotic phenomena. After 20 days, the mice were sacrificed, and the extent of pulmonary metastasis was macroscopically observed. In the present study, seleniumtreated B16F10 melanoma cells displayed a reduced MMP-2 or -9 activity, and decreased migration and invasion. Thus, the injection of selenium-treated B16F10 melanoma cells led to a lower degree of pulmonary metastasis, compared to that of the untreated B16F10 melanoma cells (Fig. 5A). In the presence of SeMet-METase an almost complete inhibition of pulmonary metastasis was evident on day 20 and most mice survived up to 60 days after tumor inoculation (Fig. 5B). 


\section{Discussion}

Recent studies suggest that chemopreventive agents, which exhibit low toxicity and are administered systematically, prevent the onset of primary melanoma and metastasis. Since methylselenol generated from organic selenium compounds, SeMet, Se-MSC or MSeA, is more bioavailable and has a lower toxicity than inorganic selenite, it takes on an increased importance as a dietary chemopreventive agent for the management of metastatic tumor cells, including melanoma $(12,13)$. A recent study has shown that methylselenol generated from SeMet $(1.25,2.5$ and $5 \mu \mathrm{M})$ and METase $(0.04 \mathrm{U} / \mathrm{ml})$ modulates the expression of MMPs and TIMP, and inhibits the migration and invasion potential of HT1080-tumor cells (34). In addition, SeMet is currently being used as the Se intervention agent in the ongoing SELECT (The Selenium and Vitamin E Cancer Prevention Trial) in the USA, and is therefore clearly regarded as safe. The safety record of selenite is, however, poor.

Despite these beneficial effects of methylselenol, limited information is currently available on the molecular targets or signaling mechanisms underlying the anti-cancer effects. We recently reported that methylselenol generated from SeMet $(25-200 \mu \mathrm{M})$ and METase $(0.02 \mathrm{U} / \mathrm{ml})$ exerts an anti-cancer effect on B16F10 melanoma cells by modulating integrin expression, altering adhesion capacity and inducing caspasemediated apoptosis (33). Integrins are the major family of cell surface receptors that mediate cell adhesion to several ECM proteins, including VN and FN. Integrin-dependent interactions with ECM proteins participate in modulating the proliferation of tumor cells and their ability to release ECMdegrading enzymes, including MMP-2, MMP-9 and uPA. Therefore, it is proposed that ECM interactions with integrins are crucial for the growth of tumors and their ability to invade and metastasize (4-9).

The neutralization of $\alpha 5$ with an anti- $\alpha 5$ antibody significantly suppresses the pulmonary metastasis potential of B16F10 melanoma cells in mice, and inhibits cell adhesion, leading to apoptosis (35). Several studies have linked the expression of $\alpha \mathrm{vB3}$ integrin with the invasion of various types of malignant tumors $(10,36,37)$. Thus, agents affecting any of these steps in association with the modulation of integrins negatively contribute to the success of metastasis, and are promising for future clinical trials.

Herein, we showed for the first time a direct correlation between a decreased integrin expression and a reduced metastatic behavior after exposure to methylselenol, through in vivo and in vitro experiments with B16F10 melanoma cells. For the consistency of in vivo experiments, B16F10 melanoma cells were treated with non-cytotoxic concentrations of SeMet (1-10 $\mu \mathrm{M})$-METase for long incubation times (48-72 h). As shown in Fig. 3B-D, SeMet-METase reduced the cell surface expression of integrin $\alpha \mathrm{V}$ and $\beta 3$ by $>50 \%$, and decreased the FN receptor expression. These, in turn, inhibited adhesion to $\mathrm{VN}$ and FN. While the specific mechanism remains unknown, it is clear that appropriate levels of integrins $\alpha \mathrm{vB3}$ and the $\mathrm{FN}$ receptors in $\mathrm{B} 16 \mathrm{~F} 10$ cells are required for metastasis. Moreover, mice inoculated with B16F10 cells treated with SeMet-METase displayed a less pulmonary metastasis than those with untreated B16F10 cells (Fig. 5A). These results are consistent with the reduction in activity of MMP-2 and -9, as well as the invasive ability (Fig. 4A-C).

In addition, we investigated the chemopreventive effects of Se-MSC, catalyzed by $ß$-lyase in the liver and kidney to produce methylselenol, by supplementation within a mash diet or drinking water. As shown in Fig. 2, a dietary oral supplementation with adequate concentrations of Se-MSC over the long term (pre- and post-tumor injection) reduced pulmonary metastasis with no toxic side-effects and consequently prolonged survival. The reduction of pulmonary metastasis and the prolonged survival of mice by supplementation of Se were also supported by increased Se levels in the tissues. The supplementation of Se-MSC from the time of tumor injection (post-tumor injection), did not induce a significant reduction in pulmonary metastasis (data not shown). In previous studies, the inhibition of pulmonary metastasis after oral supplementation with SeMet or selenite was evaluated by the inoculation of $0.5 \times 10^{5} \mathrm{~B} 16 \mathrm{~F} 10$ melanoma cells $(12,13)$. In the present study, we inoculated 6-fold more B16F10 melanoma cells $\left(3 \times 10^{5}\right)$ and demonstrated the more potent anti-metastatic effect of oral supplementation with Se-MSC.The in vivo inhibition of pulmonary metastasis by Se-MSC is not a new concept. Similarly, the in vitro inhibitory effects of methylselenol on gelatinase activities and invasion have been reported. However, the present study provides unique evidence that the decrease in integrin expression by methylselenol at non-cytotoxic concentration leads to a more potent reduction of melanoma metastasis through the inhibition of cell adhesion to ECM and a reduction in the activities of MMPs, compared to inorganic selenite. While oral supplementation with selenite inhibited pulmonary metastasis in our animal experiment (data not shown), treatment with selenite in a cell culture system did not affect integrin expression and adhesion to the ECM of B16F10 melanoma. Therefore, it may be suggested that by modulating integrin expression the inhibition of pulmonary metastasis is methylselenol-specific, but not for hydrogen selenide, which is a critical metabolite of selenite.

\section{Acknowledgements}

We thank both the Daeyu Plant Nutritional Research Institute and National Horticultural Research Institute, Rural Development Administration for Se detection in the liver, lung, serum, diet and drinking water. This work was financially supported by the Research Center for Women's Diseases of the KOSEF (R11-2005-017-03001) and by Technology Development Program for Agriculture and Forestry, Ministry of Agriculture and Forestry, Republic of Korea.

\section{References}

1. Atkins MB: The treatment of metastatic melanoma with chemotherapy and biologics. Curr Opin Oncol 9: 205-213, 1997.

2. Bialy TL, Rothe MJ and Grant-Kels JM: Dietary factors in the prevention and treatment of non-melanoma skin cancer and melanoma. Dermatol Surg 28: 1143-1152, 2002.

3. Demierre MF and Nathanson L: Chemoprevention of melanoma: an unexplored strategy. J Clin Oncol 21: 158-165, 2003.

4. Nakayama Y, Okazaki K, Shibao K, et al: Alternative expression of the collagenase and adhesion molecules in the highly metastatic clones of human colonic cancer cell lines. Clin Exp Metastasis 16: 461-469, 1998. 
5. Bafetti LM, Young TN, Itoh Y and Stack MS: Intact vitronectin induces matrix metalloproteinase-2 and tissue inhibitor of metalloproteinases-2 expression and enhanced cellular invasion by melanoma cells. J Biol Chem 273: 143-149, 1998.

6. Seftor RE, Seftor EA and Hendrix MJ: Molecular role(s) for integrins in human melanoma invasion. Cancer Metastasis Rev 18: 359-375, 1999.

7. Curran S and Murray GI: Matrix metalloproteinases: molecular aspects of their roles in tumour invasion and metastasis. Eur $\mathbf{J}$ Cancer 36: 1621-1630, 2000.

8. Morini M, Mottolese M, Ferrari N, et al: The alpha 3 beta 1 integrin is associated with mammary carcinoma cell metastasis, invasion, and gelatinase B (MMP-9) activity. Int J Cancer 87: 336-342, 2000.

9. McGary EC, Lev DC and Bar-Eli M: Cellular adhesion pathways and metastatic potential of human melanoma. Cancer Biol Ther 1 : 459-465, 2002.

10. Hosotani R, Kawaguchi M, Masui T, et al: Expression of integrin alphaVbeta3 in pancreatic carcinoma: relation to MMP-2 activation and lymph node metastasis. Pancreas 25: e30-e35, 2002.

11. Jia Y, Zeng ZZ, Markwart SM, Rockwood KF, Ignatoski KM, Ethier SP and Livant DL: Integrin fibronectin receptors in matrix metalloproteinase-1-dependent invasion by breast cancer and mammary epithelial cells. Cancer Res 64: 86748681,2004

12. Yan L, Yee JA, Li D, McGuire MH and Graef GL: Dietary supplementation of selenomethionine reduces metastasis of melanoma cells in mice. Anticancer Res 19: 1337-1342, 1999.

13. Yan L, Yee JA, McGuire MH and Graef GL: Effect of dietary supplementation of selenite on pulmonary metastasis of melanoma cells in mice. Nutr Cancer 28: 165-169, 1997.

14. Liu YH, Tian HS and Wang DX: Inhibitory effect of selenium yeast on the metastasis of Lewis lung carcinoma in C57BL mice. Studies with reference of histochemistry and ultrastructure. Chin Med J 100: 549-554, 1987.

15. Combs GF, Clark LC and Turnbull BW: Reduction of cancer risk with an oral supplement of selenium. Biomed Environ Sci 10: 227-234, 1997.

16. Blot WJ, Li JY, Taylor PR, et al: Nutrition intervention trials in Linxian, China: supplementation with specific vitamin/mineral combinations, cancer incidence, and disease-specific mortality in the general population. J Natl Cancer Inst 85: 1483-1492, 1993.

17. Clark LC and Jacobs ET: Environmental selenium and cancer: risk or protection? Cancer Epidemiol Biomarkers Prev 7: 847-852, 1998.

18. Hanada K, Satoh S, Sawamura D, Hashimoto I and Katabira Y: The inhibitory effect of selenium on mice inoculated with B16 melanoma cells. J Dermatol 13: 19-23, 1986.

19. Yeo JK, Cha SD, Cho CH, et al: Se-methylselenocysteine induces apoptosis through caspase activation and Bax cleavage mediated by calpain in SKOV-3 ovarian cancer cells. Cancer Lett 182: 83-92, 2002.

20. Redman C, Scott JA, Baines AT, et al: Inhibitory effect of selenomethionine on the growth of three selected human tumor cell lines. Cancer Lett 125: 103-110, 1998.

21. Wang Z, Jiang C, Ganther H and Lu J: Antimitogenic and proapoptotic activities of methylseleninic acid in vascular endothelial cells and associated effects on PI3K-AKT, ERK, JNK and p38 MAPK signaling. Cancer Res 61: 7171-7178, 2001.
22. Thompson HJ, Wilson A, Lu J, et al: Comparison of the effects of an organic and an inorganic form of selenium on a mammary carcinoma cell line. Carcinogenesis 15: 183-186, 1994.

23. Jiang C, Wang Z, Ganther H and Lu J: Distinct effects of methylseleninic acid versus selenite on apoptosis, cell cycle and protein kinase pathways in DU145 human prostate cancer cells. Mol Cancer Ther 1: 1059-1066, 2002.

24. Kim T, Jung U, Cho DY and Chung AS: Se-methylselenocysteine induces apoptosis through caspase activation in HL-60 cells. Carcinogenesis 22: 559-565, 2001.

25. Medina D, Thompson H, Ganther H and Ip C: Se-methylselenocysteine: a new compound for chemoprevention of breast cancer. Nutr Cancer 40: 12-17, 2001

26. Jung U, Zheng X, Yoon SO and Chung AS: Se-methylselenocysteine induces apoptosis mediated by reactive oxygen species in HL-60 cells. Free Radic Bio Med 31: 479-489, 2001.

27. Unni E, Singh U, Ganther HE and Sinha R: Se-methylselenocysteine activates caspase- 3 in mouse mammary epithelial tumor cells in vitro. Biofactors 14: 169-177, 2001

28. Jang BC, Choi ES, Im KJ, et al: Induction of apoptosis by Se-MSC in U937 human leukemia cells through release of cytochrome $\mathrm{c}$ and activation of caspases and PKC- $\delta$ : mutual regulation between caspases and PKC- $\delta$ via a positive feedback mechanism. Int J Mol Med 12: 733-739, 2003.

29. Spallholz JE, Palace VP and Reid TW: Methioninase and selenomethionine but not Se-methylselenocysteine generate methylselenol and superoxide in an in vitro chemiluminescent assay: implications for the nutritional carcinostatic activity of selenoamino acids. Biochem Pharmacol 67: 547-554, 2004.

30. Sinha R, Kiley SC, Lu JX, Thompson HJ, Moraes R, Jaken S and Medina D: Effects of methylselenocysteine on PKC activity, cdk2 phosphorylation and gadd gene expression in synchronized mouse mammary epithelial tumor cells. Cancer Lett 146: 135-145, 1999.

31. Chigbrow M and Nelson M: Inhibition of mitotic cyclin B and cdc2 kinase activity by selenomethionine in synchronized colon cancer cells. Anticancer Drugs 12: 43-50, 2001.

32. Wang Z, Jiang C and Lu J: Induction of caspase-mediated apoptosis and cell-cycle G1 arrest by selenium metabolite methylselenol. Mol Carcinog 34: 113-120, 2002.

33. Kim A, Oh JH, Park JM and Chung AS: Methylselenol generated from selenomethionine by methioninase downregulates integrin expression and induces caspase-mediated apoptosis of B16F10 melanoma cells. J Cell Physiol 212: 386-400, 2007.

34. Zeng H, Briske-Anderson M, Idso JP and Hunt CD: The selenium metabolite methylselenol inhibits the migration and invasion potential of HT1080 tumor cells. J Nutr 136: 1528-1532, 2006.

35. Qian F, Zhang ZC, Wu XF, Li YP and Xu Q: Interaction between integrin alpha(5) and fibronectin is required for metastasis of B16F10 melanoma cells. Biochem Biophys Res Commun 333: 1269-1275, 2005 .

36. Yun Z, Menter DG and Nicolson GL: Involvement of integrin alphavbeta3 in cell adhesion, motility, and liver metastasis of murine RAW117 large cell lymphoma. Cancer Res 56: 3103-3111, 1996.

37. Chatterjee $\mathrm{N}$ and Chatterjee A: Role of alphavbeta3 integrin receptor in the invasive potential of human cervical cancer (SiHa) cells. J Environ Pathol Toxicol Oncol 20: 211-221, 2001. 